learn the necessary procedure. The table shows that there is no change for the first five years, but that after 1905 the result is very evident.

Of children born 1875-1879 12 were blind from oph. neonat.

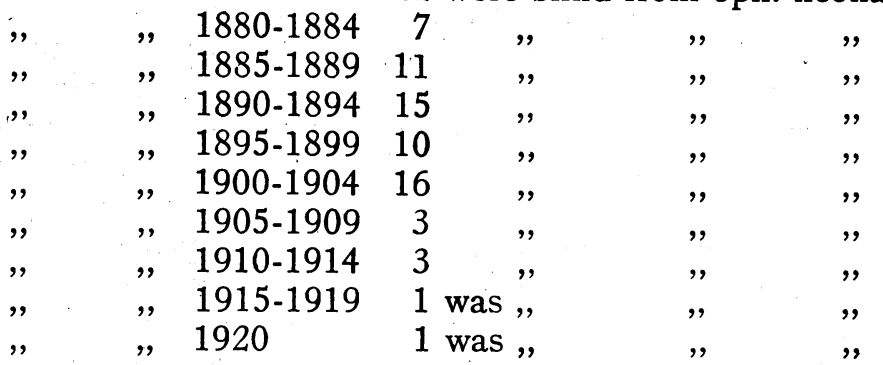

Practically all blind children born before 1916 are in the Institutes ; some children, however, born since that year may still remain in their homes, and some of these may owe their blindness to ophthalmia neonatorum.

Thanks to the excellence of our midwives our results seem to show that we may only expect one case every second year. We may possibly even improve on this, but it must be remembered that prophylactic measures cannot prevent a later infection after birth.

I may add that the population of Denmark is $3,250,000$.

\title{
ANNOTATIONS
}

\section{Standards for Out-Patient Service in Ophthalmology}

An interesting report on this subject is issued in the April number of the American Journal of Ophthalmology by the Ophthalmological section of the Associated Out-Patient Clinics of New York City. The report is divided into two sections: (1) a summary of conditions found by survey of representative eye clinics in New York; (2) an outline for an ideal out-patient service in Ophthalmology. The first part deals with the subject under the following headings: consultation facilities; medical organization; space, arrangement, equipment and facilities; admission and distribution of patients; fees ; routine history and examination; refraction and provision of glasses; follow up; records and filing; research and teaching; efficiency tests and statistics. Probably the most interesting portion of this section is that dealing with refraction, in that the reporters advise that refraction work be paid for. No ophthalmic surgeon will question the advisability of this step; it is ideal, but not always possible to obtain, at any rate in this country at the present time; nor will any deny the supreme 
importance of having the ophthalmic service linked up with other departments such as the laboratory, radiological and other departments of a general hospital. The second section of the report sets forth the principles of organization and procedure which are believed to be essential for a satisfactory out-patient service in our speciality. Consultation facilities have already been mentioned; with regard to organization and personnel, ward service and outpatient service go hand in hand; unified service is to be aimed at ; every member of the staff should have some duties and privileges in the out-patient department, and, after a proper period of probation, some ward and operating duties and privileges; the chief of clinic should be the senior assistant surgeon in the operating room, he should be in direct charge of the clinic work under the direction of the surgeon. Each new patient should be seen by the surgeon or by the chief of the clinic before a diagnosis is made or treatment instituted; the entire staff should be assigned definite hours for attendance in the out-patient department, the chief of the clinic to be present during the whole clinic session of the day on which he is on duty; clinic conferences should be held every week, and junior men should be encouraged to shew cases and make reports; lastly, the ophthalmologist should be relieved, as far as possible, of all duties not connected with the professional care of the patients. Under space, arrangement, equipment and facilities, a very complete armamentarium is devised ; in regard to procedure, it is strongly laid down that the number of patients admitted on any day should be no more than can be adequately dealt with by the staff. Much of this report is ideal ; but in this country the question of the expense is prohibitive. Most hospitals are so badly off that the governing bodies would refuse to sanction the appointment of paid refraction assistants; while, without rebuilding the whole out-patient department, it would be sometimes impossible to provide room for an adequate service such as is outlined. English ophthalmologists must, we fear, continue to carry on as best they can with the facilities provided, such as they are; it is extremely difficult to organize proper teaching when the surgeon is singlehanded and has to treat the patients without assistance, or with only inadequate assistance. Yet we feel that this report is one that should be read and studied by all connected with the management or care of an out-patient department.

\section{Mackenzie's Diseases of the Eye; Author's Copy with holograph notes}

Peculiar interest is attached to copies of any works which have been the author's own personal property, and particularly is such the case when the book in question is a famous one. No one could doubt the value of such a book as Harvey's great work on the 